\title{
Self-Supporting Graphene Films and their Applications
}

\author{
S. Goniszewski ${ }^{1,2}$, J. Gallop ${ }^{2}$, M. Adabi ${ }^{1}$, K. Gajewski ${ }^{3}$, E. Shaforost ${ }^{1}$, N. Klein ${ }^{1}$, A. Sierakowski ${ }^{4}$, J. Chen $^{5}$, \\ Y. Chen ${ }^{6}$, T. Gotszalk ${ }^{3}$ and L. Hao ${ }^{1,2}$ \\ ${ }^{1}$ Imperial College London, Department of Materials, London SW7 2AZ, UK \\ ${ }^{2}$ National Physical Laboratory, Teddington, Middlesex, TW11 OLW, UK \\ ${ }^{3}$ Wroclaw University of Technology, Faculty of Microsystem Electronics and Photonics, ul. Janiszewskiego, \\ 11/17, 50-372 Wroclaw, Poland \\ ${ }^{4}$ Institute of Electron Technology, Division of Silicon Microsystem and Nanostructure Technology, ul. \\ Pulawska 34, 05-500 Piaseczno, Poland \\ ${ }^{5}$ Department of Mechanical, Aerospace and Civil Engineering, Brunel University, Uxbridge UB8 3PH, UK \\ ${ }^{6}$ School of Information Science and Engineering, Fudan University, 20 Han Dan Road, Shanghai, \\ 200433, China
}

Corresponding author: Ling Hao e-mail: ling.hao@npl.co.uk.

Keywords: Graphene, NEMS, AFM.

\begin{abstract}
The self-supporting monolayer material which is graphene has excited enormous interest over the ten years since its discovery due to its remarkable electrical, mechanical thermal and chemical properties. In this paper we describe our work to develop chemical vapour deposition methods to grow monolayer graphene on copper foil substrates and the subsequent transfer process. Raman microscopy, scanning electron microscopy and atomic force microscopy (AFM) are used to examine the quality of the transferred material. To demonstrate the process we describe transfer onto patterned $\mathrm{SiO}_{2} / \mathrm{Si}$ substrates which forms freely suspended graphene with focus on circular wells forming graphene drums. These show interesting mechanical properties which are being explored as nanomechanical resonators.
\end{abstract}

\section{INTRODUCTION}

Graphene is a monolayer of $\mathrm{sp}^{2}$ hybridised carbon atoms and has made its way to the forefront of research and development due to its unique mechanical, electrical, thermal, optical and dimensional properties [14]. A broad spectrum of techniques have been developed to produce high quality graphene films, including mechanical exfoliation of HOPG [2], epitaxial growth from $\mathrm{SiC}[5,6]$ and, most promisingly, CVD on transition metals [7-13]. CVD graphene growth on $\mathrm{Cu}$ foils has become the most prominent method for scalable graphene growth due to its capability of producing consistent films of monolayer graphene up to tens of square metres, far surpassing any other method in scale and cost [13-16]. In this paper we first present an optimised recipe of CVD graphene growth.

CVD graphene can be transferred from transition metal substrate to any other surface. Current transfer techniques are plentiful but all include defect inducing steps and compromise either the structural integrity of the graphene or its cleanliness [17]. The majority of transfer processes apply a polymethylmethacryalate (PMMA) support layer and wet etch of the $\mathrm{Cu}$ leaving the Graphene/PMMA to be scooped onto a given substrate after being rinsed [18], this method has been proven to give reproducible and consistent results. However the physicality of this technique can lead to significant damage such as tears, cracks and wrinkles, simply from handling the delicate graphene/PMMA stack [19]. Free ions from the etchant will also be trapped in the graphene/substrate interface [18], degrading the electrical properties of the graphene via doping and scattering. The resulting chemical etch of PMMA on the surface leaves a significant amount of residue on the surface which also acts as a source of p-doping and scattering. This can be partially reduced by annealing, but high annealing temperatures $\left(300-500^{\circ} \mathrm{C}\right)$ are defect inducing in the graphene [20, 21]. Other non-etchant based methods exist such as electrochemical delamination [22], but this fails to reach the scalability needed due to its complexity. The most promising method so far is a soak-and-peel method using only hot de-ionised water to separate PMMA/Graphene from $\mathrm{Cu}$, however this method does not show easy reproducibility [23]. In this paper we present our optimised PMMA -based 
graphene transfer method that attempts to restrict all issues with PMMA -based transfer, allowing large scale CVD graphene transfer onto $\mathrm{SiO}_{2} / \mathrm{Si}$.

The high strength, breaking strain and flexibility of graphene combine to make it potentially ideal for a range of mechanical applications exemplified by suspended graphene membranes. Finally the possibility of producing free- standing CVD graphene sheets was looked at as a means of investigating the properties of graphene without the inevitable perturbations which arise from its interactions with an underlying substrate. As well as the more obvious mechanical influence from chemical and physical bonds there are also the effects of surface gating of the graphene affecting the carrier density and mobility of the material. The drums were made using the optimised CVD growth and PMMA based wet transfer processes in spite of unwanted liquid tension leading to a lesser freestanding yield. This was done due to a lower density of debris on the graphene surface compared to other support layer removal techniques such as annealing (as confirmed by AFM statistical analysis).

\section{LARGE SCALE GRAPHENE GROWTH VIA CHEMICAL VAPOUR DEPOSITION}

Graphene is grown via a chemical vapour deposition (CVD) method onto a catalyst (see Fig 1) with a surface coverage of $>95 \%$ monolayer graphene. Copper is used as the growth catalyst as opposed to other transition metals due to its low carbon solubility (established by isotope mapping of carbon 12 and carbon 13 [24]) and hence allows a self-restricting growth of single layer graphene. Successful and perfected graphene growth relies on the tuning of many variables, including; $\mathrm{Cu}$ foil pre-treatment and annealing, growth time, temperature and pressure, gas flow rates of hydrocarbon (methane $\mathrm{CH}_{4}$ ), hydrogen and buffer (Argon) gases.

FIG. 1: A cartoon depicting the mechanisms causing the growth of monolayer graphene on $\mathrm{Cu}$ during $\mathrm{CVD}$.

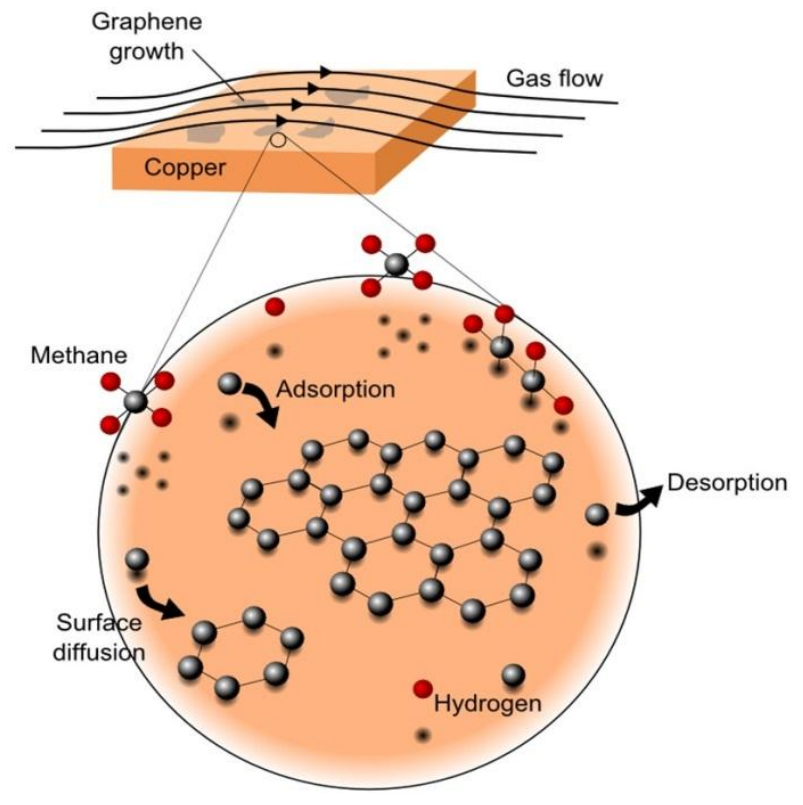

\subsection{Transition Metal Pre-Treatment}

Treating the copper foils before growth has a large effect on the graphene post CVD and it is ideal to have a flat and contaminant free copper foil for this. The copper used is a flattened $25 \mu \mathrm{m}$ thick foil of $99.95 \%$ purity. After being cut to a bulk size for growth, 1-3"x1-3" (this will be cut again for transfer), the foil is treated in acetic acid solution for 15 minutes and typically a DI water rinse is performed after, however this is omitted due to its contribution to forming a hydroxide layer on the $\mathrm{Cu}$. The $\mathrm{Cu}$ will then be annealed.

\subsection{Annealing}

Having the copper foil positioned centrally above the filament inside the CVD chamber (quartz tube), it is annealed at $1000^{\circ} \mathrm{C}$ for 60 minutes with a hydrogen flow of $30 \mathrm{sccm}$ provided by a hydrogen generator. No buffer gas such as Argon is used to keep annealing pressure low. It has been found that annealing results in larger copper grain domains. This grain growth is driven by a favourable reduction in the energy of the system caused by a reduction of the grain boundary enthalpy [25, 26]. Grain boundaries have higher available energy, therefore; grain growth is anisotropic with their growth favouring certain orientations. We have found this to be true from X-ray diffraction analysis of our pre- and post-annealed foils, see Fig 2. 


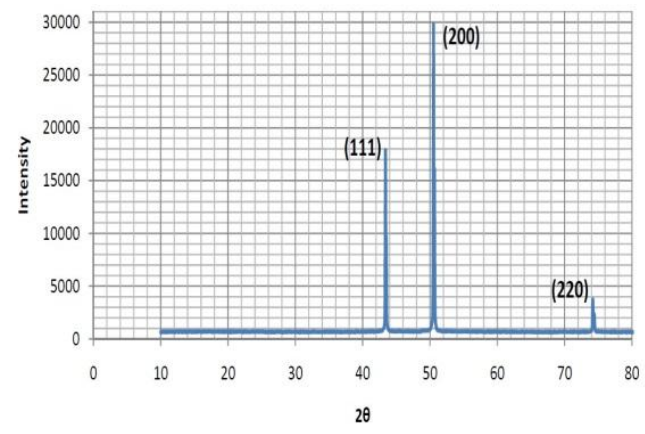

(a)

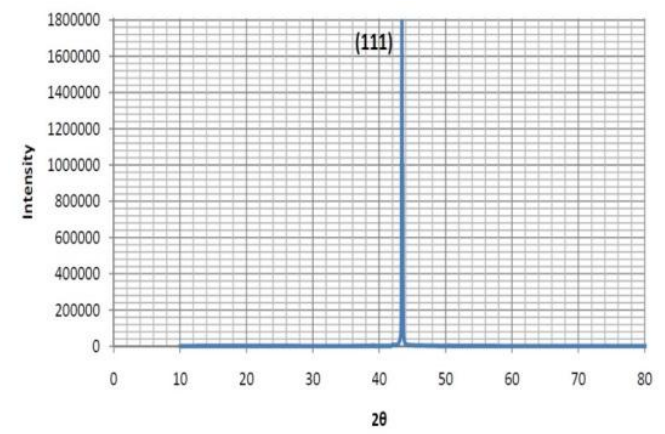

(b)

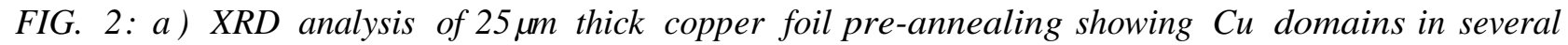

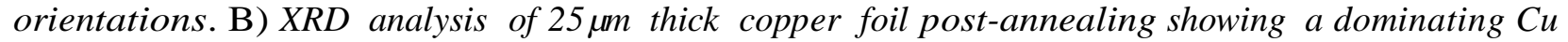
[111] orientation.

Analysing the XRD spectra, it is apparent annealing causes a shift of the relative intensity of [111] peak from $\approx 18000$ to $\approx 1800000$ and peaks associated with [200] and [220] are significantly reduced with [111] dominating. This modification of grain orientation is beneficial due to the reduction of grain boundaries per unit volume. This allows a more consistent, uninterrupted mobilisation of carbon adatoms upon the $\mathrm{Cu}$ surface during CVD. This allows larger graphene grain domains to form due to greater adatom mobilisation into existing graphene nucleations as well as a lower graphene nucleation density due to fewer regions of higher surface energy acting as nucleation sites [27].

The $\mathrm{Cu}$ we use favours [111] orientation post annealing, however we have used other $\mathrm{Cu}$ suppliers in which we see grain orientation shifts favouring other orientations when using identical annealing parameters. It can be determined that the treatment of the $\mathrm{Cu}$ by the manufacturer has a distinct effect upon the favoured orientation after annealing. This can be explained due to varying $\mathrm{Cu}$ foils having a more common grain pre-annealing. Pre-annealing the defect density is much higher, particularly grain boundary density. A larger density of grain boundaries will decrease the activation energy of grain motion due to a larger amount of stored energy; therefore more defects will lead to more probable grain growth during annealing. Another contribution of more grains is that fewer $\mathrm{Cu}$ atoms are needed to jump across a grain to cause grain boundary motion compared to coarse-grained $\mathrm{Cu}$, making grain growth more probable [28]. The relevance of this to a single grain orientation dominating is that the most common grain orientation will begin to prevail at the start of annealing when the grain growth rate is the most prominent, leading to a dominant grain orientation (but not exclusive), especially if long duration and high temperature annealing is used.

\subsection{Post Growth Cooling}

Cooling the furnace immediately after graphene growth can have a significant effect upon the resultant graphene coverage [27, 29]. Two strategies have been explored which are fast and slow cooling.

At temperatures of $700^{\circ} \mathrm{C}+$, hydrogen within the system has enough energy to bond with surface carbon to form gaseous hydrocarbon groups (reverse CVD), causing a sporadic etching of graphene, see Fig 3b. Hydrogen is needed within the system to prevent oxidation of the copper and graphene and is present from both the dissociated methane and the hydrogen flow into the system. Fast cooling takes 70 seconds to reach $700^{\circ} \mathrm{C}$ from $1035^{\circ} \mathrm{C}$ allowing a fast transition through the etching regime and gives a greater probability of continuous graphene. Slow cooling, which takes 24 times longer in our system is not favourable due to the extended period in this temperature regime with hydrogen present, leading to a higher structural defect density in the surface coverage of graphene.

\subsection{Growth Pressure and Temperature}

Under lower growth pressures, even with extended growth times, graphene typically only appears as 
separate islands and there is little continuity, see Fig 3a. This is caused by a low density of carbon adatoms on the copper surface. With higher growth pressures a larger surface coverage is seen. The cause of low surface adatom density is due to the chemical potential of methane gas. The below equation describes how the chemical potential of methane $\left(\mu_{\text {methane }}\right)$ is proportional to temperature $(T)$ and its partial gas pressure $\left(\mathrm{P}_{\text {methane }}\right)$ of the CVD gas mixture [30].

$$
\mu_{\text {methane }}=\mu_{0}+\mathrm{RT} \ln \left(\frac{\text { Pmethane }}{\text { Ptotal }}\right)
$$

Where $\mathrm{R}$ is the ideal gas constant, $\mu_{0}$ is the standard reaction enthalpy of methane and $\mathrm{P}_{\text {total }}$ is the pressure of the total gas in the system. Increasing $\mathrm{P}_{\text {methane }}$ will cause a higher chemical potential creating a surface carbon adatom density tending toward carbon supersaturation, this is due to a greater affinity between the copper substrate and carbon from the dissociated methane, thus increasing graphene domain growth.

Nucleation density saturates $\approx 4$ minutes into growth and higher temperatures correlate with lower nucleation density and larger lateral domain size of graphene. After 30 minutes the growth time has a negligible effect upon nucleation growth, even at hugely extended growth times graphene nuclei will not merge unless the temperature threshold of $1000^{\circ} \mathrm{C}$ is met, leaving channels of exposed $\mathrm{Cu}$, see Fig 3c. Our optimised growth recipe for our CVD system uses a pressure of $110 \mathrm{mbar}$ and growth temperatures $1035^{\circ} \mathrm{C}$.

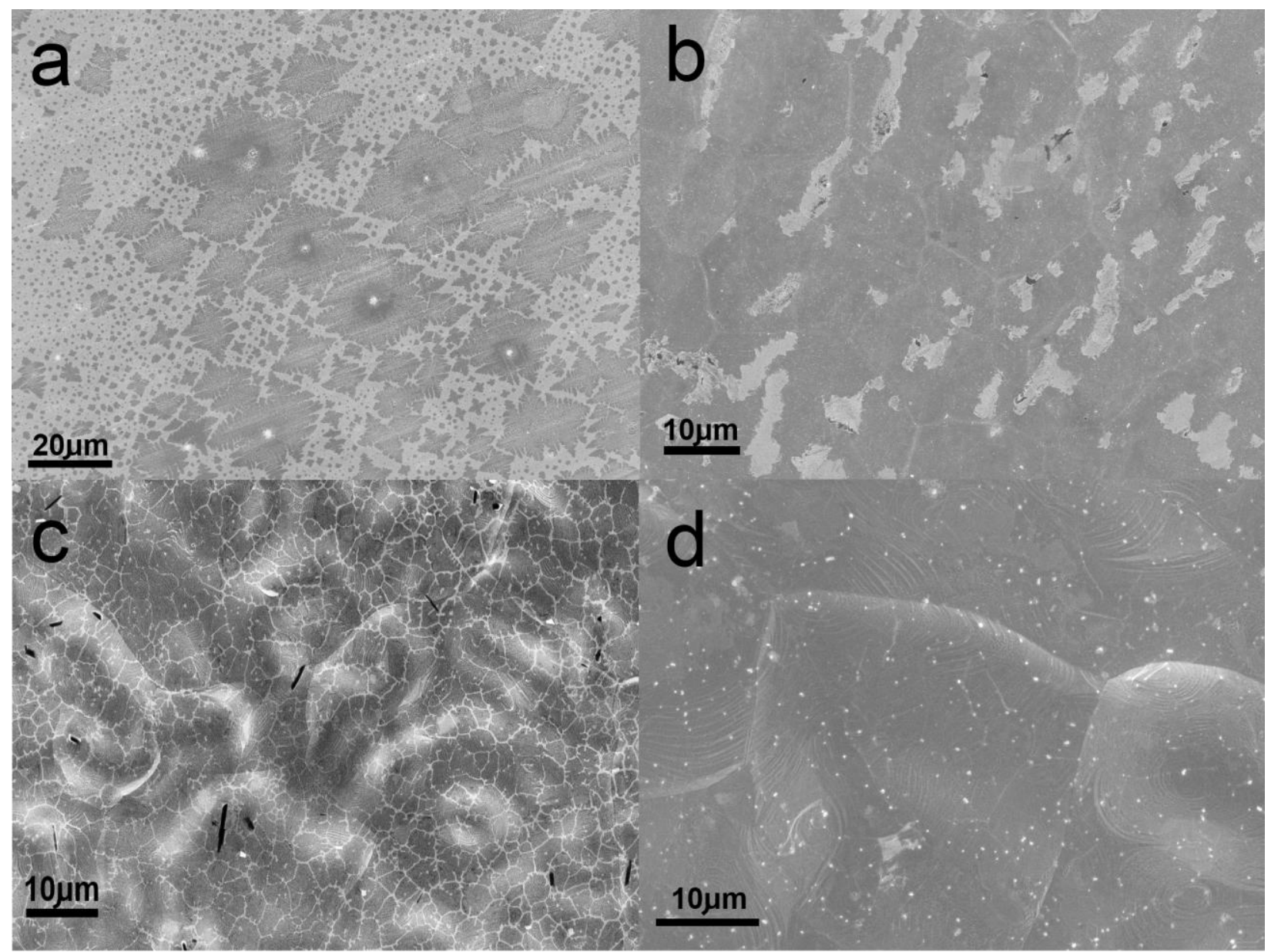

FIG. 3: SEM images of graphene and subsequent artefacts of growth from different growth parameters. a.) Inconsistent growth of graphene leaving separate nucleations and little continuous graphene due to low pressure growth (2mbar). b.) Blotchy areas of no graphene caused by reverse CVD. This is due to hydrogen etching of graphene and is prominent during slow cooling when the system is at $700^{\circ+} \mathrm{C}+$ for extended periods of time. c.) Low temperature growth $\left(900^{\circ} \mathrm{C}\right)$ of graphene showing a reluctance of graphene nucleations to merge with each other even at extended growth times of over 120 minutes. $d$.) High surface 
coverage monolayer graphene using temperature of $1035^{\circ} \mathrm{C}$, pressure of $110 \mathrm{mbar}$, methane flow rate of 1 sccm and hydrogen flow rate of $80 \mathrm{sccm}$.

\subsection{Methane Flow Rate}

The domain size and density of graphene nucleations is largely dependent upon methane flow rates. Methane flow rates of $\geq 1 \mathrm{sccm}$ are used and give continuous surface coverage and have been established as the standard for the system used as even this low flow rate supplies a critical density of surface carbon adatoms. A methane flow rate of $\geq 1 \mathrm{sccm}$ does not always give a full coverage and it is possible a larger flow rate may be more beneficial to domain size, however broken surface coverage in these circumstances is likely to be due to other effects such as low pressure, low temperature, hydrogen etching or methane dilution with buffer gases causing higher pressures but lower methane density per volume.

Monolayer graphene with small sites of bi/multi-layer on the $\mathrm{Cu}$ surface can be grown using methane flow of $1 \mathrm{sccm}$ with short growth times of 12 minutes $/ \mathrm{cm}^{2}$ with $\mathrm{H}_{2}$ flow rates of $80 \mathrm{sccm}$, see Fig $3 \mathrm{~d}$.

\section{GRAPHENE TRANSFER}

Graphene is transferred onto patterned $\mathrm{Si} / \mathrm{SiO}_{2}$ substrates using a PMMA support layer method. Giving a high quality yield of freestanding graphene structures (graphene drums) over the patterns etched into the substrate. $\mathrm{SiO}_{2}$ is used as the substrate surface due to its dielectric properties and strong adhesion/van der Waals forces with monolayer graphene $\left(0.45+/-0.02 \mathrm{~J} / \mathrm{m}^{2}\right)$ [31]. This force is large in comparison to most in micromechanical structures; this importantly provides strong anchoring for the graphene drums when brought to mechanical resonance. High quality CVD graphene transfer via this method is highly influenced by several variables including; substrate and substrate treatment, polymer support layer, transition metal etchant, cleaning of the sample, polymer removal and handling.

\subsection{Making the substrate}

A $\mathrm{Si}$ substrate with a thermally grown $300 \mathrm{~nm}$ thick $\mathrm{SiO}_{2}$ layer was patterned by optical photolithography with a large array of shapes including circles and squares with diameters ranging from 2-50 $\mu \mathrm{m}[32]$. The photomask includes an array of navigational features, with target crosses and respective row and column numbering to allow location of each shape. Wet etching was used to remove the oxide so that the patterns represented an array of $1-3 \mu \mathrm{m}$ deep depressions across the Si chip. The etched wafers were cut into $10 \mathrm{~mm} \times 10 \mathrm{~mm}$ chips each containing four repeats of the overall pattern, each one occupying a quadrant of the chip.

\subsection{Substrate Treatment}

The treatment of substrates is key to optimising graphene transfer. $\mathrm{SiO}_{2} / \mathrm{Si}$ substrates are a standard for graphene transfer and their treatment will alter graphene's electrical properties extensively. Oxygen plasma and UV ozone treatments are used to clean the substrate surface but cause a degree of hydrophilicity, creating a dipolar molecule affinity at the surface due to oxidation [33]. Due to graphene's field effect behaviour, this will result in the shifting of the Dirac point to varying levels of p-doping caused by the local surface charge density (proportional to hydrophilicity) which can be determined using surface contact angle measurements. These results will be discussed in further detail in another publication.

\subsection{Graphene Transfer Process}

The graphene transfer process is as follows, see Fig 4: Liquid PMMA (950 PMMA A6, 6\% of polymethyl-methacrylate in Anisole) is placed onto the CVD grown graphene via a syringe $(0.1 \mathrm{ml}$ PMMA per $\mathrm{cm}^{2}$ graphene) then spin-coated at 8000RPM for 60 seconds, creating a PMMA layer of thickness $\leq 400 \mathrm{~nm}$. The sample is cured under ambient conditions (fume cupboard in non-clean room lab) for 24 hours allowing the PMMA to naturally relax onto graphene rather than be forced to 
cure which has a greater chance of inducing defects such as tears in the graphene. The CVD process grows graphene on the top and bottom of $\mathrm{Cu}$ due to gas flow underneath the $\mathrm{Cu}$ during $\mathrm{CVD}$, this can also be caused by carbon precipitating out to all surfaces upon cooling after diffusion of carbon species into the $\mathrm{Cu}$ foil during CVD, however this will be minimal due to the low carbon solubility of $\mathrm{Cu}[13,24,27]$. The uncoated (no PMMA) side of $\mathrm{Cu}$ is treated by floating it on a bath of $10 \%$ nitric acid solution (HNO3) for one minute to remove the unwanted graphene that has grown on the bottom side of the $\mathrm{Cu}$ foil, leaving a $\mathrm{Cu} / \mathrm{Graphene/PMMA} \mathrm{stack}$. This is done to prevent the monolayer of graphene on the bottom side of $\mathrm{Cu}$ contacting the top side graphene once the $\mathrm{Cu}$ has been removed, which would create a bi-layer post $\mathrm{Cu}$ etch.

The PMMA/graphene/Cu sample is then placed in a copper etchant, an ammonium persulphate solution $\left(1 \mathrm{~g}\left(\mathrm{NH}_{4}\right)_{2} \mathrm{~S}_{2} \mathrm{O}_{8}\right.$ per $100 \mathrm{ml}$ ultra-pure $18.2 \mathrm{M} \mathrm{cm}$ at $25^{\circ} \mathrm{C}$ water), a series of three etchant exposures is used, each up to 12 hours. $\left(\mathrm{NH}_{4}\right)_{2} \mathrm{~S}_{2} \mathrm{O}_{8}$ has been found to be a superior $\mathrm{Cu}$ etchant leaving little residue compared to other commonly used etchants such as Ferric Chloride $\left(3.5 \mathrm{~g} \mathrm{FeCl}_{3}\right.$ in $100 \mathrm{ml}$ water) which leaves significant amounts of iron on the graphene surface which needs post treatment with Hydrochloric Acid $(\mathrm{HCl})$. Following $\mathrm{Cu}$ etching, two ultra-pure water baths are used to rinse the sample by floating it on the liquid surface for up to 6 hours per bath. The sample is moved between baths and etchants using a small beaker to scoop the sample and some of the liquid and is then poured into the new solution, the graphene is constantly sat on a small amount of liquid. This slightly contaminates the new bath with the liquid the graphene is sat on from the previous bath, but the amount of contamination is negligible in comparison to the new volume of etchant/rinse as confirmed with $\mathrm{pH}$ testing. This technique prevents the graphene contacting the glass or transfer scoop which can easily damage the graphene by transferring it in part to the surface it touches.

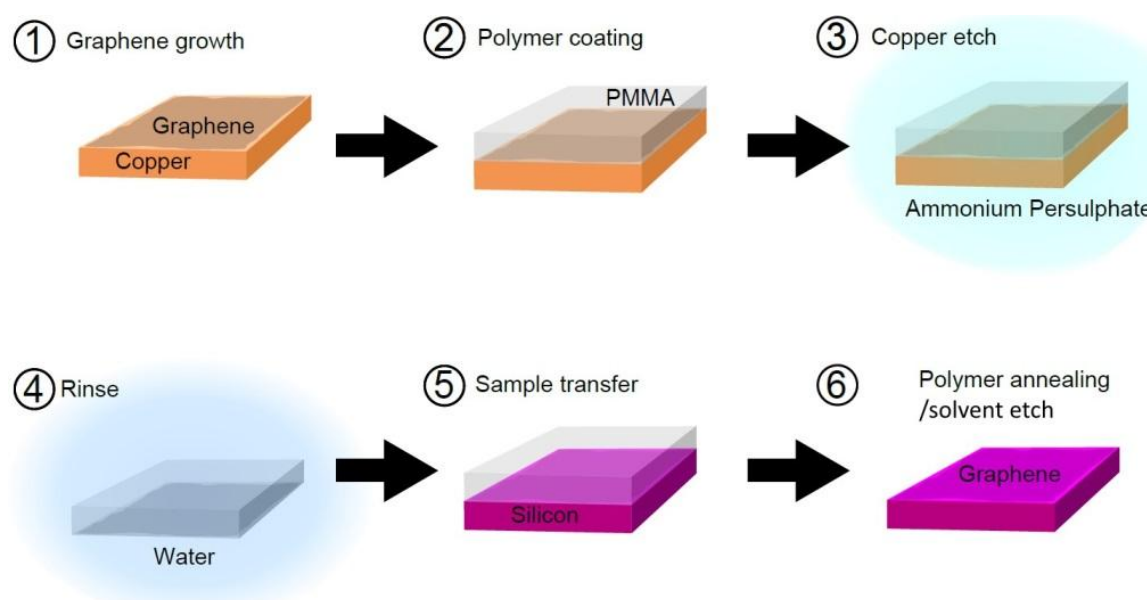

\section{FIG. 4: A simplified drawing of the wet graphene transfer process onto substrates for both freestanding and flat graphene.}

The sample is then scooped onto the wanted substrate by approaching the sample perpendicularly to the substrate, a gentle contact between the two is made and then the substrate is pulled out, connecting the sample to the surface with minimal wrinkling.

\subsection{Removing PMMA}

For optimum mechanical resonator performance the PMMA/Graphene stack is ironed out via heating the PMMA, this softens and expands PMMA allowing it to relax flat upon the substrate, minimising any wrinkling of the graphene and in turn restricts mechanical and electrical degradation. The heating process also serves to anneal the sample, removing PMMA via hydrogenolysis and depolymerisation. The conditions for annealing are $350^{\circ} \mathrm{C}$ in a furnace for 10 hours at $\approx 2 \mathrm{mbar}$ with a hydrogen flow rate of $100 \mathrm{sccm}$. Argon is omitted from the process as it causes a dilution of hydrogen, leading to a direct increase in polymer debris left on the surface of graphene as cutting of -carboxyl functionalization is 
diminished. This also allows for a capillary effect of PMMA vapour due to insufficient dilution causing debris build up in the features of patterned substrates.

Wet solvent etching of the PMMA gives a cleaner surface in comparison to annealing but due to surface tension effects gives a much smaller yield of complete freestanding graphene structures, for graphene flush against an unpatterned substrate this is the optimum PMMA etch technique. Dichloromethane (DCM) is used to etch structures for 24 hours in a light current created by a magnetic stirrer, the DCM is then replaced with Isopropanol (IPA). The IPA is rapidly heated, significantly lowering its surface tension and it is allowed to evaporate away without boiling. This gives comparable results to using a critical point dryer as outlined in [34].

\section{RAMAN ANALYSIS}

Perfect graphene exhibits Raman peaks at D (1350/cm), G(1580/cm) and 2D (2690/cm) [35-37]. The graphene we have grown shows all of these defining peaks, see Fig 5. The D band is strongly associated with disorder (it is an intervalley phonon scattering from $\mathrm{K}$ to $\mathrm{K}^{\prime}$ points) and subsequently an increase in intensity of $\mathrm{I}(\mathrm{D}) / \mathrm{I}(\mathrm{G})$ ratios also indicates an increase in disorder. We've found that graphene on the $\mathrm{SiO}_{2}$ surface shows a strong D band while it is negligible in freestanding graphene, it can therefore be speculated that the interaction with the $\mathrm{SiO}_{2}$ is disorder inducing. Other peaks such as D + G $(2940 / \mathrm{cm})$ and D' $(1620 / \mathrm{cm})$ are also indicative of a level of disorder in the graphene but do not present themselves strongly in our graphene Raman spectra. The $\mathrm{I}(\mathrm{D}) / \mathrm{I}(\mathrm{G})$ peak ratio shows two separate behaviours with disorder increase, at lower defect density the ratio will increase proportionally due to a greater quantity of elastic scattering which again reinforces the hypothesis that the substrate is disorder inducing as we have no existing ratio in freestanding graphene due to the lack of $\mathrm{D}$ band. As graphene disorder increases to a point where graphene can be defined to be amorphous carbon the ratio will begin to decrease due to an attenuation of all graphene defining bands, we do not see such an attenuation in any of our samples.

With an increase in graphene layers additional forces are present due to interactions between the stacked layers. With greater layer number a splitting of the 2D peak will be seen along with an increased wavenumber, reduced intensity and reduced FWHM. The G peak is also affected by layer number and will red shift with increasing layers. Therefore an assumption of layer number can be derived by taking the ratio of $2 \mathrm{D}$ and $\mathrm{G}$ peak intensities $(\mathrm{I}(2 \mathrm{D}) / \mathrm{I}(\mathrm{G}))[36,37]$. A poor ratio is measured in our $\mathrm{SiO}_{2}$ bonded graphene of $\approx 1$ whereas the freestanding graphene on the same sample has a ratio averaging at 1.5 (with maximums close to 5) which is very close to the optimum of 2. These results suggest that the number of graphene layers increases over the $\mathrm{SiO}_{2}$, however this is not the case as confirmed with AFM and is an artifact from the substrate interaction with graphene. A possible explanation for this is hydrocarbons/free radicals are trapped between graphene and the $\mathrm{SiO}_{2}$ as a result of the transfer process and the carbon/doping effects from these give an impression of bi/multi-layer graphene.

A shift in Fermi level from the Dirac point is a consequence of doping in the graphene and corresponds to a decrease in probability of excited charge carrier recombination [38]. This causes perturbations of photons to be non-adiabatic, removing the Kohn anomaly (an anomaly in the dispersion relation of metals at points of high symmetry in the first Brillouin zone, causing the energy of a specific phonon to be lowered) and increasing the phonon energy for the G peak, increasing its frequency and decreasing its FWHM [39]. With increasing electron concentration a decrease in 2D peak position can be seen due to a hypothesized expansion of the crystal lattice directly reducing the energy of the characterizing phonons [39]. Oddly we see very close values to the accepted graphene peaks in our $\mathrm{SiO}_{2}$ bonded graphene whereas the freestanding graphene has red-shifted values for both $G$ and 2D peaks, see table 1. This suggests that our freestanding graphene is doped while the $\mathrm{SiO}_{2}$ bonded graphene is not. This is highly doubtful and it is likely that the blueshift of the bonded graphene corresponds to the substrate p-doping the graphene while graphene is un-doped while freestanding at the lower wavenumber values. The total shift away from normal wavenumber for both freestanding and bonded graphene is possibly an artefact from laser power and wavelength $(532 \mathrm{~nm})$ used. 


\begin{tabular}{|c|c|c|c|c|}
\hline Peak & $\begin{array}{c}\text { Freestanding } \\
\text { Wavenumber }(/ \mathbf{c m})\end{array}$ & $\begin{array}{c}\text { Peak FWHM } \\
\text { Freestanding }\end{array}$ & $\begin{array}{c}\text { On } \mathrm{SiO}_{2} \\
\text { Wavenumber } \\
(/ \mathbf{c m})\end{array}$ & $\begin{array}{c}\text { Peak FWHM On } \\
\mathrm{SiO}_{2}\end{array}$ \\
\hline 2D & 2638.94 & 8.75675 & 2665.2 & 15.0576 \\
\hline $\mathrm{G}$ & 1568.58 & 6.20261 & 1579.22 & 6.49305 \\
\hline $\mathrm{D}$ & $\mathrm{N} / \mathrm{A}$ & $\mathrm{N} / \mathrm{A}$ & 1339.5 & 6.72014 \\
\hline $\mathrm{I}(2 \mathrm{D}) / \mathrm{I}(\mathrm{G})$ & 1.5 & $\mathrm{~N} / \mathrm{A}$ & 1 & $\mathrm{~N} / \mathrm{A}$ \\
\hline $\mathrm{I}(\mathrm{D}) / \mathrm{I}(\mathrm{G})$ & $\mathrm{N} / \mathrm{A}$ & $\mathrm{N} / \mathrm{A}$ & 0.4 & $\mathrm{~N} / \mathrm{A}$ \\
\hline
\end{tabular}

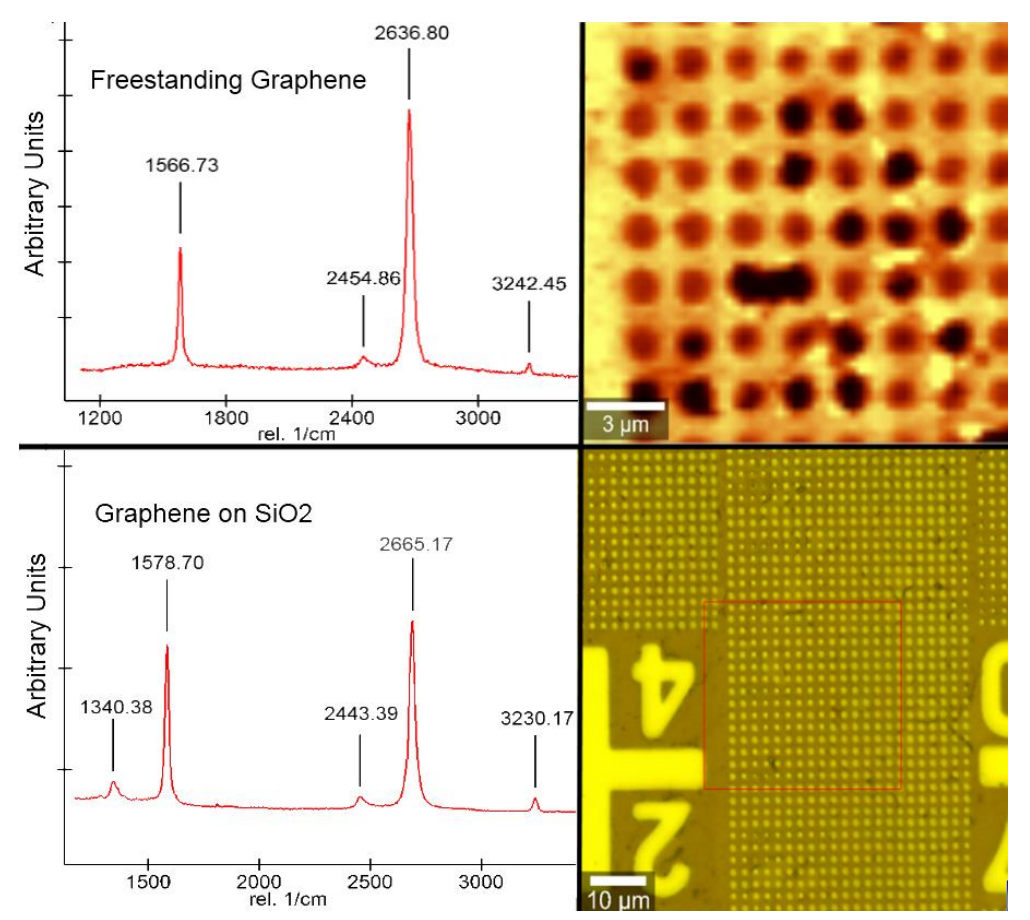

FIG. 5: Top left and Bottom left, Raman spectra of the average peaks of freestanding graphene and graphene bonded to $\mathrm{SiO} 2$ respectively. Top right, Raman representation of $2 \mathrm{D}$ peak intensity of graphene sample showing clear areas of freestanding graphene drums (circular areas of dimmer brightness), graphene on SiO2 (lighter areas) and areas of no graphene (black areas). Bottom right, optical image of the Raman scan area.

\begin{tabular}{|c|c|c|c|c|}
\hline Peak & $\begin{array}{c}\text { Freestanding } \\
\text { Wavenumber }(/ \mathrm{cm})\end{array}$ & $\begin{array}{c}\text { Peak FWHM } \\
\text { Freestanding }\end{array}$ & $\begin{array}{c}\text { On SiO } \\
\text { Wavenumber } \\
(/ \mathbf{c m})\end{array}$ & $\begin{array}{c}\text { Peak FWHM On } \\
\mathrm{SiO}_{2}\end{array}$ \\
\hline 2D & 2638.94 & 8.75675 & 2665.2 & 15.0576 \\
\hline $\mathrm{G}$ & 1568.58 & 6.20261 & 1579.22 & 6.49305 \\
\hline $\mathrm{D}$ & $\mathrm{N} / \mathrm{A}$ & $\mathrm{N} / \mathrm{A}$ & 1339.5 & 6.72014 \\
\hline $\mathrm{I}(2 \mathrm{D}) / \mathrm{I}(\mathrm{G})$ & 1.5 & $\mathrm{~N} / \mathrm{A}$ & 1 & $\mathrm{~N} / \mathrm{A}$ \\
\hline $\mathrm{I}(\mathrm{D}) / \mathrm{I}(\mathrm{G})$ & $\mathrm{N} / \mathrm{A}$ & $\mathrm{N} / \mathrm{A}$ & 0.4 & $\mathrm{~N} / \mathrm{A}$ \\
\hline
\end{tabular}

Table 1. The wavenumber Raman peak values for D, $G$ and $2 D$ for freestanding and SiO2 bonded graphene and their corresponding FWHM and intensity ratios.

\section{AFM ANALYSIS OF DRUM SHAPE}

Optical and AFM images of the chip with transferred graphene have been recorded. Optical images 
are capable of showing the presence of graphene films, particularly as the $\mathrm{SiO}_{2}$ layer on the $\mathrm{Si}$ is $300 \mathrm{~nm}$, a thickness which is chosen to give good interference colour contrast [40]. It is also possible to see that some depressions are not covered by graphene, others are partially covered. Also, interestingly some graphene membranes sag into the depressions, appearing to touch the bare Si base. AFM images confirm this behaviour quantitatively. Some suspended graphene membranes show different behaviour. In these cases the AFM images show a bulge rather than sagging. The AFM line scans across the centre of a $2 \mu \mathrm{m}$ diameter hole and a $5 \mu \mathrm{m}$ square which a single graphene layer is stretched in the transfer process is shown in Fig 6 and 7 respectively.
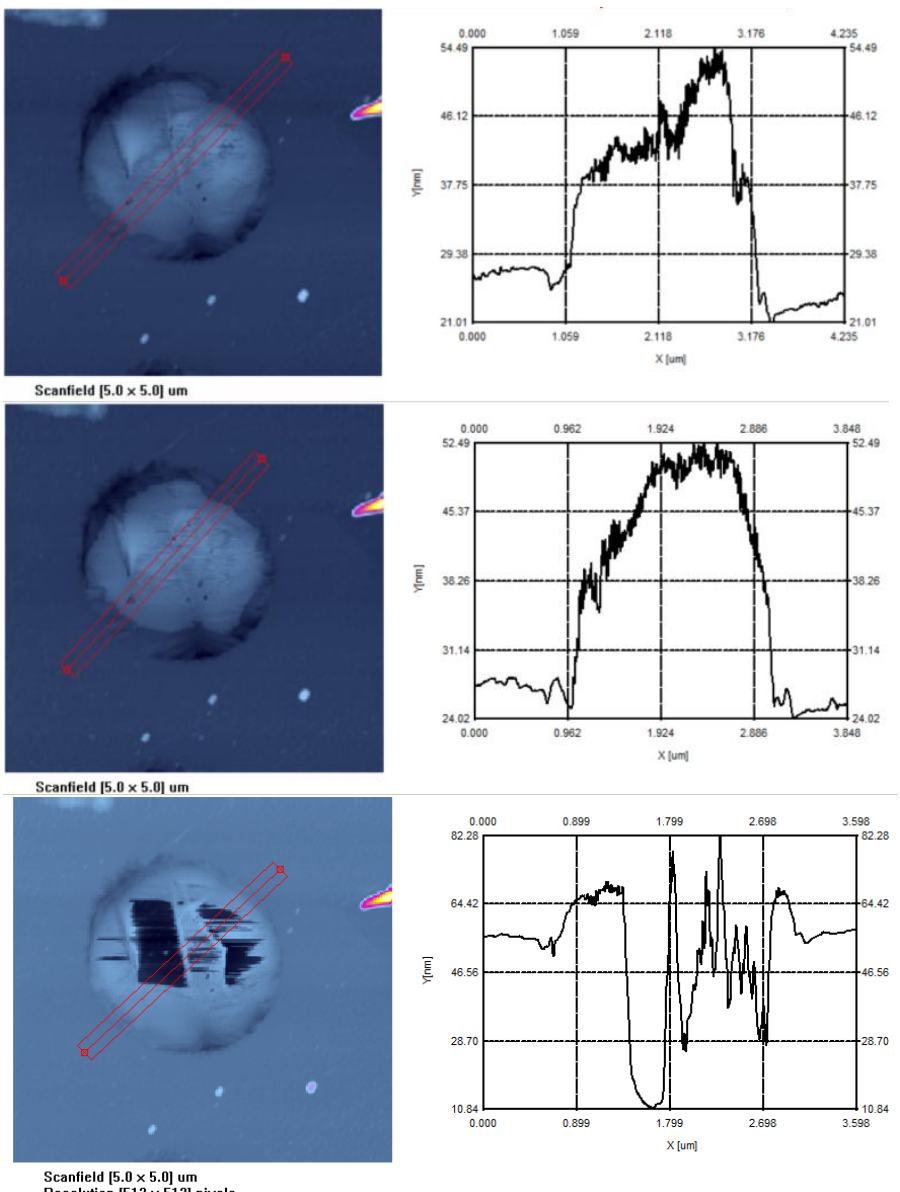

FIG. 6: AFM line scans across a $2 \mu \mathrm{m}$ diameter graphene drums. In the sequence of images the AFM set-point is successively increased. The bulge in the membrane increases at first but then in the final image the membrane goes slack.
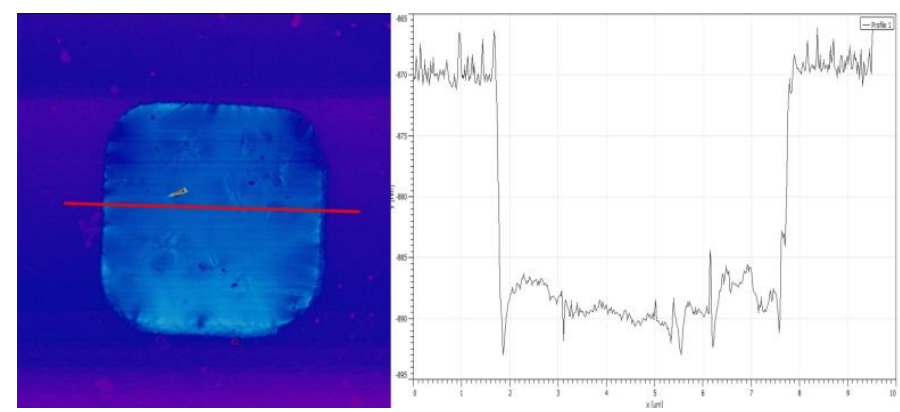

FIG. 7: AFM line scan across a 5um diameter graphene square. 
It is interesting to try to fit the measured shape of the membrane to a simple model of excess pressure on the constrained side of the graphene. Note that it has been observed that graphene is surprisingly impermeable to all gases with the exception of water vapour (or even liquid water) [41]. Equation 2 shows the total force on the membrane of radius $\mathrm{R}$ with excess pressure $\Delta \mathrm{p}$ across it and is pictured in Fig 8.

$$
F=\Delta p \pi R^{2}=2 \pi \sigma t R \sin (\alpha)
$$

Here $\sigma$ is the stress in the membrane which is related to the radial strain $\varepsilon$ and the membrane Young's modulus $\mathrm{E}$ by the expression

$$
\sigma=\frac{\varepsilon E}{1-v^{2}}
$$

where $v$ is Poisson's ratio for graphene and is believed to be approximately equal to 0.2 .

We ignore the residual stress $\sigma_{0}$ and assume only that arising from Hooke's law since any residual stress would cause even higher values of $\Delta \mathrm{p}$. The result of the central bulge by an amount $\Delta \mathrm{z}(0)$ allows us to calculate the additional length of the parabolic shaped membrane if we know the tangential angle $\alpha$ at its circumference. For small deflections $(\Delta z(0)<<R) \sin (\alpha) \approx \Delta z(0) / R$ allowing us to derive the final expression for $\Delta p$.

$$
\Delta p=\frac{8 \Delta z(0)^{3} E t}{3 R^{4}\left(1-v^{2}\right)}
$$

Consider the AFM line-scan shown in Fig 6. For now we ignore the internal stress. The displacement of the graphene membrane centre $\Delta \mathrm{z}(0)$ is some $25 \mathrm{~nm}$ above the level of the Si wafer surface. The radius $R$ is $1 \mu \mathrm{m}$, the membrane thickness $\mathrm{t}$ is $0.4 \mathrm{~nm}$ since the graphene is shown to be predominantly single layer via Raman analysis in Section 4. Finally the Youngs modulus $E$ has values in the range 0.3 to $1.0 \mathrm{~T}$ $\mathrm{Pa}$ for graphene. Even assuming the lower of these values the pressure difference $\Delta$ p required to produce such a bulge is around 0.2 atmospheres which is not realistic given that all transfer and processing is done at room pressure and temperature. The fact that some membranes sag also indicates that there is not an excess pressure within the wells.

We tentatively rule out this as a possible source of the convex parabolic bulge of the membranes. Instead we regard the observed shape of the membranes as arising from the attractive (van der Waals) force between the AFM tip and the surface of the membrane. This explanation is supported by a scan of the same area with reduced separation between the AFM tip and the surface (achieved by changing the AFM control electronics set point). Now the bulge at the centre of the membrane above the substrate is increased, which would not happen if the bulge was only due to excess pressure. Further increase of the AFM set point (i.e. closer contact and therefore higher attractive force) leads to a dramatic change in shape on scanning. Now the bulge disappears and the shape of the membrane becomes ill-defined. We believe this may be explained by the increasing attractive force between AFM tip and membrane becoming sufficient to overcome the adhesive force between the vertical wall of the well and the graphene membrane which had been drawn into it. When the force is sufficient to pull the membrane up and away from the vertical wall the diameter of the membrane becomes greater than the diameter of the hole and therefore the membrane is no longer constrained and becomes flexibly positioned, as shown in the lowest image of Fig 6. On reversing this process and reducing the force between membrane and AFM tip the van der Waals forces become sufficient to draw the graphene membrane in again to the vertical wall of the hole, leading to a return to the original bulging shape. This explanation also is supported by some observations we have made on mechanical resonances of these membranes. We have measured the piezo excited mechanical resonances using a novel near-field microwave readout method [42,43]. These resonances show modest quality factors (Q) in air due, we believe, to air damping. These results show that, as the piezo drive force increases, non-linear behaviour is observed. Unlike most other mechanical systems, the non-linearity leads to softening of the membrane i.e. the resonant frequency reduces with 
increased drive level. This could again be interpreted as the effective radius of the membrane being increased as the side-wall length is reduced at higher drive levels. We will describe this effect in more detail elsewhere.

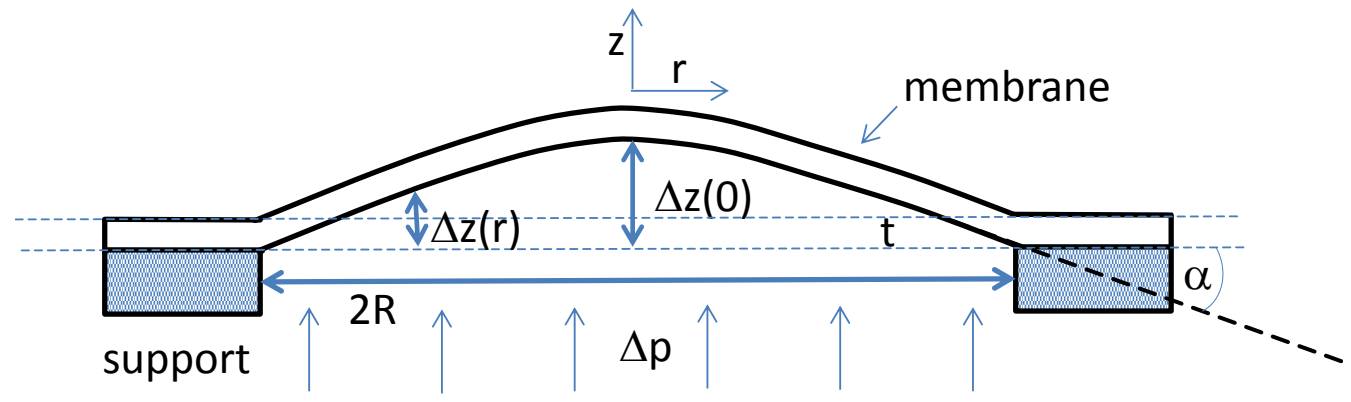

FIG. 8: Schematic of shape of thin membrane under the influence of excess pressure $\Delta p$.

\section{CONCLUSIONS}

Graphene is a remarkable material in many different ways. In this paper we demonstrate that a waferscale growth technique, chemical vapour deposition, followed by a polymer based wet transfer method is capable of allowing large areas (several square $\mathrm{mm}$ ) to be transferred onto a substrate giving freely suspended graphene membranes with relatively high yield of intact resonators. We show that these can be non-destructively imaged by AFM and can be manipulated by the same scanned technique. Furthermore these membrane resonators show an unexpected non-linear behaviour which is explained by the sagging of unstrained graphene into the supporting well shapes. The remarkable resilience of self-supporting single atomic layers over microns of distance reinforces the belief that graphene mechanical resonators can provide exceptional sensors for example detection of single molecule adsorbed masses. Since the areal mass of single layer graphene is so extremely small and frequency is such an accurately measured parameter the frequency shift produced by adsorption of a single molecule on a graphene drum should be readily detectable, even at room temperature. This should lead to a significant range of useful applications in biology and more widely in sensitive detection.

\section{ACKNOWLEDGEMENTS}

This work was supported by the UK NMS Programme, the EU EMRP (European Metrology Research Programme) projects MetNEMS and GraphOhm. The EMRP is jointly funded by the participating countries within EURAMET and the European Union.

\section{REFERENCES}

[1] Ovid'ko, I.: 'Mechanical properties of graphene', Reviews on Advanced Materials Science, 2013, 34, pp. $1-11$

[2] Geim, A., Novoselov, K.: 'The rise of graphene', Nature materials, 2007, 6, (3), pp. 183-191

[3] Novoselov, K. S ., Fal, V., Colombo, L., et al.: 'A roadmap for graphene', Nature, 2012, 490, (7419), pp. 192-200

[4] Balandin, A. A., Ghosh, S., Bao, W., et al.: 'Superior thermal conductivity of single-layer graphene', Nano letters, 2007, 8, (3), pp. 902-907

[5] Virojanadara, C., Zakharov, A., Yakimova, R., et al.: 'Buffer layer free large area bi-layer graphene on sic (0001)',Surface Science, 2010, 604, (2), pp. L4-L7

[6] Robinson, J., Hollander, M., LaBella, M., et al.: 'Epitaxial graphene transistors: enhancing performance via hydrogen intercalation', Nano letters, 2011, 11, (9), pp. 3875-3880

[7] Iwasaki, T., Park, H., Konuma, M., et al.: 'Long-range ordered single-crystal graphene on high-quality heteroepitaxial ni thin films grown on mgo (111)', Nano letters, 2010, 11, (1), pp. $79-84$ 
[8] Reina, A., Jia, X., Ho, J., et al.: 'Large area, few-layer graphene films on arbitrary substrates by chemical vapor deposition', Nano letters, 2008, 9, (1), pp. 30-35

[9] Li, X., Magnuson, C., Venugopal, A., et al.: 'Graphene films with large domain size by a two-step chemical vapor deposition process', Nano letters, 2010, 10, (11), pp. 4328-4334

[10] Celebi, K., Cole, M., Teo, K., et al.: 'Observations of early stage graphene growth on copper',

Electrochemical and Solid-State Letters, 2011, 15, (1), pp. K1-K4

[11] Li, X., Magnuson, C., Venugopal, A., et al.: 'Large-area graphene single crystals grown by lowpressure chemical vapor deposition of methane on copper', Journal of the American Chemical Society, 2011, 133, (9), pp. 2816-2819

[12] Bae, S., Kim, H., Lee, Y., et al.: 'Roll-to-roll production of 30-inch graphene films for transparent electrodes', Nature nanotechnology, 2010, 5, (8), pp. 574-578

[13] Li, X., Cai, W., An, J., et al.: 'Large-area synthesis of high-quality and uniform graphene films on copper foils', Science, 2009, 324, (5932), pp. 1312-1314

[14] Wood, J. D., Schmucker, S.W., Lyons, A. S., et al.: 'Effects of polycrystalline cu substrate on graphene growth by chemical vapor deposition', Nano letters, 2011,11, (11), pp. 4547-4554

[15] Kim, K. S., Zhao, Y., Jang, Y., et al.: 'Large-scale pattern growth of graphene films for stretchable transparent electrodes', Nature, 2009, 457, (7230), pp. 706-710

[16] Baker, J.: 'Graphene at Manchester: The route to commercialisation', Graphene \& 2D Materials Conference: From Research to Applications 2014, National Physical Laboratory, Teddington, UK, November 2014 .

[17] De Arco, L. G., Zhang, Y., Kumar, A., et al.: 'Synthesis, transfer, and devices of single-and few-layer graphene by chemical vapor deposition', Nanotechnology, IEEE Transactions on, 2009, 8, (2), pp. 135-138

[18] Li, X., Zhu, Y., Cai, W., et al.: 'Transfer of large-area graphene films for high-performance transparent conductive electrodes', Nano letters, 2009, 9, (12), pp. 4359-4363

[19] Suk, J. W., Kitt, A., Magnuson, C.W., et al.: 'Transfer of cvd-grown monolayer graphene onto arbitrary substrates', ACS nano, 2011, 5, (9), pp. 6916-6924

[20] Ahn, Y., Kim, H., Kim, Y. -H., et al.: 'Procedure of removing polymer residues and its influences on electronic and structural characteristics of graphene', Applied Physics Letters, 2013, 102, (9), p. 091602

[21] Kumar, K., Kim, Y. -S., Yang. E. -H., et al.: 'The influence of thermal annealing to remove polymeric residue on the electronic doping and morphological characteristics of graphene', Carbon, 2013, 65, pp. 35-45

[22] Wang, Y., Zheng, Y., Xu, X., et al.: 'Electrochemical delamination of cvd-grown graphene film: toward the recyclable use of copper catalyst', ACS nano, 2011, 5, (12), pp. 9927-9933

[23] Gupta, P., Dongare, P. D., Grover, S., et al.: 'A facile process for soak-and-peel delamination of cvd graphene from substrates using water', Scientific reports, 2014, 4,

[24] Li, X., Cai, W., Colombo, L., et al.: 'Evolution of graphene growth on ni and cu by carbon isotope labeling', Nano letters, 2009, 9, (12), pp. 4268-4272

[25] Gertsman, V. Y., Birringer. R.:'On the room-temperature grain growth in nanocrystalline copper', Scripta metallurgica et materialia, 1994,30, (5), pp. 577-581

[26] Hoehne, K., Sizmann. R.:'Volume and surface self-diffusion measurements on copper by thermal surface smoothing', physica status solidi (a), 1971, 5, (3), pp. 577-589

[27] Mattevi, C., Kim, H., Chhowalla. M., 'A review of chemical vapour deposition of graphene on copper', Journal of Materials Chemistry, 2011, 21, (10), pp. 3324-3334

[28] Simoes, S., Calinas, R., Vieira, M., et al.: 'In situ tem study of grain growth in nanocrystalline copper thin films', Nanotechnology, 2010, 21, (14), p. 145701

[29] Bhaviripudi, S ., Jia, X., Dresselhaus, M. S., et al.: 'Role of kinetic factors in chemical vapor deposition synthesis of uniform large area graphene using copper catalyst', Nano letters, 2010, 10, (10), pp. 4128-4133

[30] Carlsson, J. -O., Martin. P.: 'Chemical vapor deposition', Handbook of Deposition Technologies for Films and Coatings: Science, Applications and Technology, 3rd ed., edited by PM Martin (Elsevier Inc., Oxford, 2010), 2010, p. 406 
[31] Koenig, S. P., Boddeti, N. G., Dunn, M. L., et al.: 'Ultrastrong adhesion of graphene membranes', Nature nanotechnology, 2011, 6, (9), pp. 543-546

[32] Gajewski, K., Gotszalk, T., Sierakowski, A., et al.: 'Microfabricated support structures for investigations of mechanical and electrical graphene properties', Electron Technology Conference 2013, International Society for Optics and Photonics., 2013 pp. 89020G-89020G

[33] Vig. J. R.: 'Ultraviolet-ozone cleaning of semiconductor surfaces', 1992, Technical report,

DTIC Document

[34] Zande, A. M. v. d., Barton, R. A., Alden, J. S., et al.: 'Large-scale arrays of single-layer graphene resonators', Nano letters, 2010, 10, (12), pp. 4869-4873

[35] Berciaud, S., Ryu, S., Brus, L. E., et al.: 'Probing the intrinsic properties of exfoliated graphene: Raman spectroscopy of free-standing monolayers', Nano letters, 2008, 9, (1), pp. 346-352

[36] Ferrari, A., Meyer, J., Scardaci, V., et al.: 'Raman spectrum of graphene and graphene layers', Physical review letters, 2006, 97, (18), p. 187401

[37] Ferrari. A. C.: 'Raman spectroscopy of graphene and graphite: disorder, electron-phonon coupling, doping and nonadiabatic effects', Solid state communications, 2007, 143, (1), pp. 4757

[38] Pisana, S., Lazzeri, M., Casiraghi, C., et al.: 'Breakdown of the adiabatic born-oppenheimer approximation in graphene', Nature materials, 2007, 6, (3), pp. 198-201

[39] Das, A., Pisana, S., Chakraborty, B ., et al.: 'Monitoring dopants by raman scattering in an electrochemically top-gated graphene transistor', Nature nanotechnology, 2008, 3, (4), pp. 210-215

[40] Blake, P., Hill, E., Neto, A. C., et al.: 'Making graphene visible', Applied Physics Letters, 2007, 91(6), p. 063124

[41] Nair, R., Wu, H., Jayaram, P., et al.: 'Unimpeded permeation of water through helium-leaktight graphene- based membranes', Science, 2012, 335, (6067), pp. 442-444

[42] Hao, L., Gallop, J., Chen. J.:'Near-field microwave excitation and detection of nems resonators', Nanotechnology (IEEE- NANO), 2012 12th IEEE Conference on, 2012 , pp. 1-5

[43] Hao, L., Goniszewski, S., J. Chen., et al.: 'Microwave excitation and readout of nano-and micron scale cantilevers',Applied Surface Science, 2012, 258, (6), pp. 2192-2195 